\title{
PEACEFUL APPLICATIONS OF NUCLEAR FISSION
}

A SYMPOSIUM on the "Peaceful Applications of Nuclear Fission" was arranged by Section A (Mathematics and Physics) at the recent meeting of the British Association at Dundee. It was opened by the president of the Section, Sir Edward Appleton, who said that no scientific subject had ever aroused quite the same mixture of hopes and fears. At that meeting they were to concentrate on the hopes; in due course the work conducted on the development of the atomic bomb would lead to the saving of far more lives than were lost in the attacks on Japan.

Dr. J.D. Cockcroft remarked that the peace-time applications of nuclear fission would result from the achievement of a divergent nuclear chain reaction in the atomic pile-first built by Prof. E. Fermi in the University of Chicago in 1942. The fission pile provides a much more powerful source of neutrons than the largest cyclotron. The pile would make possible new types of experiments in nuclear physics and would be the main source of radioactive isotopes. With the production of neutrons goes the development of heat, and the pile is a potential though not certain new source of power for the world.

The first objective of the Atomic Energy Research Establishment at Harwell, Berks, had been the construction of a low-power graphite pile known as "G.L.E.E.P." similar to that now in use at the Argonne National Laboratory in Chicago. This pile consists of several hundred tons of graphite bricks between which are grooves for the insertion of uranium metal and uranium oxide. Surrounding the pile is a shield of conorete about $5 \mathrm{ft}$. in thickness. This is required to reduce the intensity of the neutron and gamma radiation developed inside the pile to tolerance-levels. The shield is pierced with holes for control instruments, control rods, safety rods and for 'thermal columns' to bring neutrons of thermal energy out to the shield face for experiments.

The concrete shield and its accessories took about a year to build. Loading of the uranium started on August 5 and was completed by August 15 of this year. During the loading of the uranium the multiplication constant, $k$, of the pile increased towards unity, and since one neutron released in the spontaneous fission of a U238 nucleus leads to $1+k+k^{2}$ or $1 /(1-k)$ neutrons, the course of approach to the critical or divergent state is followed by recording by a boron trifluoride-filled counter the flux of neutrons inside the pile. Thus on August 7, when 7 tons of uranium had been loaded, the counter recorded 17 neutrons per minute. By August 11 this had increased to 55 neutrons/minute, by the morning of August 15 to 2,400 and by early afternoon to 6,600 per minute. At this point the counter was saturated-the pile being almost divergent. During the last stages of loading, the control rods were partially inserted, reducing $k$ slightly. Uranium was then loaded until the divergent point was just passed. At this stage the pile generated about 0.1 watt of thermal energy. The power-level increases exponentially with time but with a very long period. The period depends on $1 /(k-1)$, and with an excess of $k$ of $1 / 40,000$ the period is one hour. The pile is therefore very easy to control. A withdrawal of the control rods led to a decrease of the period to about a minute and the power-level was allowed to increase to 100 watts, at which level ionization chambers had been pre-set to release the shut-off rods and shut down the pile. The first objective of the Harwell work had, therefore, been realized in about fifteen months.

Measurements would now be carried out to determine all the pile characteristics. This was expected to take about one month. After that the power-level would be increased to a level set by the safe operating temperature of the uranium. In this pile only very simple cooling is used, and it would probably be necessary to limit the average power to about 50 kilowatts. At this power-level there would be a maximum thermal neutron flux of $10^{10}$ neutrons/sq.cm./sec.; the total number of neutrons generated would be about $3 \times 10^{15}$ per sec., of which about one per cent could be used for production of radioactive isotopes or for experimental work. It was intended to start radioactive isotope production immediately. Some isotopes could be produced in adequate intensities by this pile-thus it is estimated that 5-10 curies per month of radiophosphorus could be produced monthly by the capture of neutrons in phosphorus. Production of high speeific activity $\mathrm{P}^{32}$ by the $(n, p)$ reaction in sulphur would be considerably smaller. Long-lived isotopes such as $\mathrm{C}^{14}$ could only be produced in small quantities, and adequate production would have to await the completion of the second Harwell pile.

One of the principal applications of the low-power pile is the study of the nuclear reactions of elements with slow neutrons. The introduction of as little as $0.1 \mathrm{mgm}$. of cadmium into the pile produces a detectable change of power-level ; and from the rate of change, the absorption cross-section for s!ow neutrons can be determined. In practice, specimens under investigation are moved into and out of the pile with a period of 20 sec. or so and the amplitude of oscillation of the power-level measured by a resonant galvanometer or other suitable methods. A pile of this type is an indispensable tool for the control of nuclear properties of all basic materials used in the construction of piles.

The power-level of a pile can be increased by more intensive cooling of the uranium metal, and in the second Harwell pile the metal rods will be cooled by a high-speed stream of air flowing past the rods. In this way the power-level could be increased to several thousand kilowatts. The uranium metal would be sheathed with aluminium to prevent oxidation and escape of fission products. The decreasing creep strength of aluminium with increasing temperature would probably limit surface temperatures to about $350^{\circ} \mathrm{C}$, , and emergent gas temperatures would therefore be limited to about $300^{\circ} \mathrm{C}$. Although this is not high enough for efficient power production, a heat exchanger would be placed in the emerging gas stream and some use made of the pile heat.

The emergent gas temperature could be increased if a material having more favourable proporties could be substituted for aluminium. Metals such as steel would reduce the multiplication constant below unity ; beryllium would be suitable if it could be produced in ductile form.

The second Harwell pile will be used to study problems of power production. Thus it is important to study the effect of neutron irradiation on the 
physical properties of materials used in piles-it is known that some deterioration takes place. It is also important to study the effect of formation of fission products on the multiplication constant of the pile, and in particular the effect of the partial substitution of U235 by plutonium in the pile.

It is expected that the neutron flux and available neutrons will be of the order of a hundred times that available in the G.L.E.E.P. This would make possible the full production of most radioactive isotopes required in Britain. A few isotopes would be limited in quantity.

This pile would also provide a powerful source of fission products - which are distributed among the thirty-five elements from zinc to gadolinium. At $1,000 \mathrm{~kW}$. power-level, about a gram a day of fission products would be produced.

Other types of pile important for scientific research include the heavy-water piles. These piles have a higher multiplication constant than the graphite pile, and would use a substantially smaller amount of uranium and moderator. For a fixed power output they could give a much higher neutron flux, and this is very important for some experiments and for the study of future developments. A high-power heavy-water pile has recently been completed at Chalk River by the Canadian National Research Council, and will provide an important experimental tool for atomic energy research.

The future of the application of nuclear fission to the large-scale development of power is at present obscured by lack of information on a number of essential points.

A pile using natural uranium sheathed by aluminium and cooled by a circulating gas could be used for steam generation and power production by a steam turbine. Experimental plants of this type would probably be built, though their thermodynamic efficiency would be low, due to the temperature limit of aluminium. If Nature were exceptionally kind, a plant of this kind containing 100 tons of uranium would generate $100,000 \mathrm{~kW}$. of thermal energy for twenty years without fuel replacement. Actually, however, the formation of fission products will probably lead to poisoning of the chain reaction due to their additional absorption, and difficulties may be experienced due to deterioration of materials under the intense neutron bombardment of the pile. The metal will, therefore, have to be withdrawn at frequent intervals, the fission products extracted and the uranium and plutonium reprocessed. Until operating experience tells us how frequently this will have to be done, and what the costs will be, it is not possible to predict the costs of nuclear power. The position about reserves of nuclear fuel is equally uncertain at the present time, since the proportion of the uranium which can be used is unknown.

So far, piles have used only a fraction of the energy of U235. In the future, we may hope to do considerably better than this. As a pile burns up the primary fuel atoms of U235, the noutrons released in the fission can turn U238 into plutonium, which is fissionable by slow neutrons and is a secondary fuel just as good as U235. One U235 atom will in general be replaced by rather less than one plutonium atom this in turn will be partially replaced. The overall result is that there is a possibility of utilizing more than the fission energy of U235. Ideally we might go a long way towards reaching the full utilization of U238-how far we can go we do not know.
There is also a possibility of utilizing the fission energy of thorium by turning it into U233, which is also fissionable by slow neutrons.

One other major uncertainty stands in the way. With nuclear power goes a very large-scale production of radioactive materials. A power plant generating the thermal energy of a million kilowatts produces daily an activity of the order of a million curies even after an appreciable decay is allowed for. A world run on nuclear power would generate at least a hundred times this activity. The safe disposal of these products presents a major problem.

Prof. O. R. Frisch described the application of the fission pile to nuclear research. A high-power pile of the type being built at Harwell would produce very large numbers of fast and slow neutrons. Experiments requiring fast neutrons continue to be carried out, for the most part, on cyclotrons, which provide a point source of fast neutrons, whereas the pile neutrons are spread out over several thousand cubic feet of pile volume. The pile has, however, a decisive advantage for experiments with slow neutrons, since the cyclotron source becomes diffuse in slowing down the neutrons. Experimental work was described using a mechanical velocity selector to study the velocity distribution of slow neutrons emerging from the pile. Filtering through graphite has been shown to reduce their mean velocity by a factor of 4 owing to their wave properties leading to selective transmission through the graphite lattice. This is due to the absence, for very slow neutrons, of lattice planes fulfilling the Bragg reflexion condition. On the other hand, homogeneous neutron beams can be obtained by Bragg reflexion from crystals, and the crystal spectrometer provides a powerful method of studying neutron-capture phenomena. It is expected also that neutron scattering from hydrogen atoms in crystals may result in neutron spectroscopy being a valuable tool for the organic chemist. Slow neutrons have been shown to be totally reflected at glancing angles; this proves that the refractive index of the materials used (glass, graphite, etc.) is less than unity for neutron waves. The intense beam of pile neutrons would make it worth while to look for the decay of the neutron into a proton and an electron.

Mr. W. G. Marley described the production of radioactive isotopes in a pile. The simplest procedure is to produce them by neutron capture, that is, by $(n, \gamma)$ reactions. The materials to be irradiated are placed in the reflector of the pile, where up to onefortieth of the pile neutrons can be absorbed for this purpose.

Some biological experiments require a high specific activity -500 millicuries per gram of materials. For this purpose it might be necessary to use $(n, p)$ reactions, for example, in $\mathrm{S}^{32}(n, p) \mathrm{P}^{32}$. Many of these reactions require fast neutrons and can only be produced in the core of the pile, where the available neutrons will be only about one-tenth of those available in the reflector. A few isotopes such as tritium would be produced by $(n, \alpha)$ reactions. The highpower Harwell pile would be able to produce quantities of most isotopes more than adequate for all likely requirements. A few long-lived isotopes such as tritium, C14, Cl37, would be limited by pile capacity, though an annual production of 4-5 euries of C14 should be possible.

It is expected that a few radioactive isotopes, such as $\mathrm{Na} 22$, will still have to be produced in the cyclotron, 
since they can only be produced by neutrons by $(n, 2 n)$ reactions requiring very fast neutrons.

Radioactive isotopes produced by $(n, p)$ reactions would require chemical separation; and when the processes have been established at the Harwell station, routine separation will be carried out by the Radiochemical Centre of the Ministry of Supply. The allocation of radioactive isotopes will be carried out by a sub-committee of the Advisory Council for the Radiochemical Centre. This sub-committee, under the chairmanship of Dr. J. D. Cockcroft, includes representatives of user interests - the Medical and Agricultural Research Councils and the Department of Scientific and Industrial Research, which will represent the universities and industry.

Radioactive isotopes from piles in the United States have been available to American users only since 1946, and a large number of applications to medical, biological and industrial research have already been found, while the future has been declared by Dr. G. Seaborg to hold unlimited possibilities.

\section{J. D. CockCrofT}

\section{OBSERVATIONS ON THE TRACKS OF SLOW MESONS IN PHOTO- GRAPHIC EMULSIONS*}

\author{
By C. M. G. LATTES, DR. G. P. S. OCCHIALINI \\ and DR. C. F. POWELL
}

H. H. Wills Physical Laboratory, University of Bristol

\begin{abstract}
TNTRODUCTION. In recent experiments, it has been shown that charged mesons, brought to rest in photographic emulsions, sometimes lead to the production of secondary mesons. We have now extended these observations by examining plates exposed in the Bolivian Andes at a height of 5,500 m., and have found, in all, forty examples of the process leading to the production of secondary mesons. In eleven of these, the secondary particle is brought to rest in the emulsion so that its range can be determined. In Part 1 of this article, the measurements made on these tracks are described, and it is shown that they provide evidence for the existence of mesons of different mass. In Part 2, we present further evidence on the production of mesons, which allows us to show that many of the observed mesons are locally generated in the 'explosive' disintegration of nuclei, and to discuss the relationship of the different types of mesons observed in photographic plates to the penetrating component of the cosmic radiation investigated in experiments with Wilson chambers and counters.
\end{abstract}

\section{Part I. Existence of Mesons of Different Mass}

As in the previous communications ${ }^{1}$, we refer to any particle with a mass intermediate between that of a proton and an electron as a meson. It may be emphasized that, in using this term, we do not imply

- This article contains a summary of the main features of a number of lectures given, one at Manchester on June 18 and four at the Conference on Cosmic Rays and Nuclear Physics, organised by Prof W. Heitler, at the Dublin Institute of Advanced Studies, July 5-12. A complete account of the observations, and of the conclusions which A complete account of the observations, and of that the corresponding particle necessarily has a strong interaction with nucleons, or that it is closely associated with the forces responsible for the cohesion of nuclei.

We have now observed a total of 644 meson tracks which end in the emulsion of our plates. 451 of these were found, in plates of various types, exposed at an altitude of $2,800 \mathrm{~m}$. at the Observatory of the Pic du Midi, in the Pyrenees; and 193 in similar plates exposed at $5,500 \mathrm{~m}$. at Chacaltaya in the Bolivian Andes. The 451 tracks in the plates exposed at an altitude of $2,800 \mathrm{~m}$. were observed in the examination of 5 c.c. emulsion. This corresponds to the arrival of about 1.5 mesons per c.c. per day, a figure which represents a lower limit, for the tracks of some mesons may be lost through fading, and through failure to observe tracks of very short range. The true number will thus be somewhat higher. In any event, the value is of the same order of magnitude as that we should expect to observe in delayed coincidence experiments at a height of $2,800 \mathrm{~m}$., basing our estimates on the observations obtained in similar experiments at sea-level, and making reasonable assumptions about the increase in the number of slow mesons with altitude. It is therefore certain that the mesons we observe are a common constituent of the cosmic radiation.

Photomicrographs of two of the new examples of secondary mesons, Nos. III and IV, are shown in Figs. 1 and 2. Table 1 gives details of the character. istics of all events of this type observed up to the time of writing, in which the secondary particle comes to the end of its range in the emulsion.

TABLR 1

Event No.

Range in emulsion in microns of Primary meson Secondary meson

$\begin{array}{rr}133 & 613 \\ 84 & 565 \\ 1040 & 621 \\ 133 & 591 \\ 117 & 638 \\ 49 & 595 \\ 460 & 616 \\ 900 & 610 \\ 239 & 666 \\ 256 & 637 \\ 81 & 590\end{array}$

Mean range $614 \pm 8 \mu$. Straggling coefficient $\sqrt{\bar{\Sigma} \Delta_{i}^{2} / n}=4 \cdot 3$ per cent, where $\Delta_{i}=R_{i}-\bar{R}, R_{i}$ being the range of a secondary meson, and $\bar{R}$ the mean value for $n$ particles of this type.

The distribution in range of the secondary particles is shown in Fig. 3. The values refer to the lengths of the projections of the actual trajectories of the particles on a plane parallel to the surface of the emulsion. The true ranges cannot, however, be very different from the values given, for each track is inclined at only a small angle to the plane of the emulsion over the greater part of its length. In addition to the results for the secondary mesons which stop in the emulsion, and which are represented in Fig. 3 by black squares, the length of a number of tracks from the same process, which pass out of the emulsion when near the end of their range, are represented by open squares.

\section{The $\mu$-Decay of Mesons}

Two important conclusions follow from these measurements. Our observations show that the directions of ejection of the secondary mesons are orientated at random. We can therefore calculate the probability that the trajectory of a secondary 